\title{
2-Phenitidine derivatives as suitable inhibitors of butyrylcholinesterase
}

\author{
Muhammad Athar Abbasi,",, Aziz-ur-Rehman', Muhammad Zahid Qureshi', Farhan Mehmood \\ Khan $^{1}$, Khalid Mohmmed Khan², Muhammad Ashraf ${ }^{3}$, Iftikhar Afzal ${ }^{4}$
}

\begin{abstract}
${ }^{1}$ Department of Chemistry, Government College University, Lahore, Pakistan, ${ }^{2}$ HEJ Research Institute of Chemistry, International Center for Chemical and Biological Sciences, University of Karachi, Karachi, Pakistan, ${ }^{3}$ Department of Biochemistry and Biotechnology, ${ }^{4}$ Department of Pharmacy, The Islamia University of Bahawalpur, Bahawalpur, Pakistan
\end{abstract}

\begin{abstract}
This manuscript reports the synthesis of a series of $N$-substituted derivatives of 2-phenitidine. First, the reaction of 2-phenitidine (1) with benzene sulfonyl chloride (2) yielded $N$-(2-ethoxyphenyl) benzenesulfonamide (3), which further on treatment with sodium hydride and alkyl halides (4a-g) furnished into new sulfonamides (5a-g). Second, the phenitidine reacted with benzoyl chloride (6) and acetyl chloride (8) to yield the reported $N$-benzoyl phenitidine (7) and $N$-acetyl phenitidine (9), respectively. These derivatives were characterized by infrared spectroscopy, ${ }^{1} \mathrm{H}-\mathrm{NMR}$, and EI-MS, and then screened against acetylcholinesterase, butylcholinesterase, and lipoxygenase enzyme, and were found to be potent inhibitors of butyrylcholinesterase alone.
\end{abstract}

Uniterms: 2-Phenitidine/inhibitor activity. Sulfonamides. Acetamide. Benzamide. Butyrylcholinesterase.

\begin{abstract}
Este trabalho apresenta a síntese de uma série de derivados da 2-fenetidina $N$-substituídos. Primeiro, a reação da 2 -fenetidina (1) com cloreto de benzenossulfonila (2) conduziu à $N$-(2-etoxifenil) benzenossulfonamida (3) que, após tratamento com hidreto de sódio e haletos de alquila (4a-g), originou novas sulfonamidas (5a-g). Em segundo lugar, a reação da fenetidina com cloreto de benzoíla (6) e cloreto de acetila (8) conduziu, respectivamente, à $N$-benzoilfenetidina (7) e $N$-acetilfenetidina (9). A caracterização destes derivados fez-se por IV, ${ }^{1} \mathrm{H}-\mathrm{RMN}$ e EM-IE. Procedeu-se à avaliação da atividade inibidora destes compostos em relação às enzimas acetilcolinesterase, butirilcolinesterase e lipoxigenase. No entanto, apenas revelaram atividade inibidora da butirilcolinesterase.
\end{abstract}

Unitermos: 2-Fenetidina/atividade inibidora. Sulfonamidas. Acetamida. Benzamida. Butirilcolinesterase.

\section{INTRODUCTION}

Compounds bearing the $-\mathrm{SO}_{2}-\mathrm{NH}$ - group (the sulfonamide group) have long been known to be potent inhibitors of carbonic anhydrase (CA) (Remko et al., 2010; Supuran et al., 2003), and are widely used as antibacterial agents. Synthesis of sulfonamides involves the nucleophilic attack of ammonia or amine on a sulfonyl halide (Chan et al., 2002; Shaabani et al., 2007). On the other hand, arylsulfonyl azides can be reduced to convert into arylsulfonamides (Boruah et al., 1997). It is also known

\footnotetext{
*Correspondence: Muhammad Athar Abbasi. Department of Chemistry, Government College University, Lahore-54000, Pakistan. E-mail: atrabbasi@yahoo.com
}

that aryl sulfonamides act as antitumor agents through the perturbation of the cell cycle in the G1 phase, during angiogenesis inhibition or distribution of the microtubule assembly (Saczewski et al., 2008; Garaj et al., 2005; Saczewski et al., 2006; Supuran et al., 2003). Various sulfonamides have also been reported to act as antitumor agents through carbonic anhydrase (CA) inhibition (Supuran et al., 2003; Supuran et al., 2004; Kivela et al., 2005; Supuran et al., 2007).

Acetylcholinesterase (AChE, EC 3.1.1.7) and butyrylcholinesterase (BChE, EC 3.1.1.8) consist of a family of enzymes, which include serine hydrolases. The different specificities for the substrates and the inhibitors for these enzymes are due to the differences in the amino acid residues of the active sites of AChE and BChE. The enzyme 
system is responsible for the termination of acetylcholine at the cholinergic synapses. These are key components of cholinergic brain synapses and neuromuscular junctions. The major functions of $\mathrm{AChE}$ and $\mathrm{BChE}$ are to catalyze the hydrolysis of the neurotransmitter acetylcholine, and to terminate the nerve impulse at the cholinergic synapses (Cygler et al., 1993; Tougu et al., 2001). It has been found that BChE (E.C 3.1.1.8) inhibition is an effective tool for the treatment of Alzheimer's disease and related dementias. BChE is found in significantly higher quantities in Alzheimer's plaques than in plaques found among normal age-related brains without dementia. $\mathrm{BChE}$ is produced in the liver and enriches blood circulation. In addition, it is also present in adipose tissue, and can also be found in the intestine, smooth muscle cells, white matter of the brain, and in many other tissues (Gauthier et al., 2001). Therefore, the search for new cholinesterase inhibitors is considered an important and ongoing strategy in the introduction of new drug candidates for the treatment of Alzheimer's disease and other related diseases (Bertaccini et al., 1982).

In this work, we report on the various $N$-substituted derivatives of 2-phenitidine. First, a new parent sulfonamide [ $N$-(2-ethoxyphenyl)benzenesulfonamide] was prepared by reacting benzenesulfonyl chloride with 2-phenitidine at room temperature in excellent yield. Simple stirring in basic media gave the desired compound that was further processed to obtain different new $N$-alkyl substituted sulfonamides, and then 2-phenitidine was also acylated with benzoyl chloride and acetyl chloride, which have already been reported (Downer, Jackson, 2007; Smith et al., 2003).

A survey of the literature revealed that slight modifications in the structure can result in qualitative as well as quantitative changes in activity, which prompted us to undertake the synthesis of various 2-phenitidine derivatives and to study their structure-activity relationship by screening them against DPPH, AChE, BChE, and lipoxygenase (LOX), and these were found to be active against $\mathrm{BChE}$, and were also possible entrants for the treatment of Alzheimer's disease.

\section{MATERIALS AND METHODS}

\section{General}

Thin-layer chromatography (TLC) was performed on pre-coated silica gel G-25-UV ${ }_{254}$ plates. Detection was carried out at $254 \mathrm{~nm}$ with a ceric sulphate reagent. Purity was checked on TLC with different solvent systems using ethyl acetate and $n$-hexane, giving a single spot. The infra- red (IR) spectra were recorded in $\mathrm{KBr}$ on a Jasco-320-A spectrophotometer (wave number in $\mathrm{cm}^{-1}$ ). ${ }^{1} \mathrm{H}-\mathrm{NMR}$ spectra were recorded in $\mathrm{CDCl}_{3}$ on a Bruker spectrometer operating at $400 \mathrm{MHz}$. Chemical shifts are given in ppm. Mass spectra (EI-MS) were recorded on a JMS-HX-110 spectrometer with a data system. The melting points were recorded on a Griffin and George melting point apparatus by open capillary tube, and these were uncorrected.

\section{Procedure for the synthesis of sulfonamide in aqueous media}

The nucleophilic substitution reaction of amine with benzene sulfonyl chloride was carried out as follows: a mixture of benzenesulfonyl chloride $(10.0 \mathrm{mmol}$; $1.27 \mathrm{~mL})$ and 2-phenitidine (10.0 mmol; $1.43 \mathrm{~mL})$ was suspended in $25 \mathrm{~mL}$ of water. The $\mathrm{pH}$ of the suspension was adjusted and was maintained at 9.0 by adding a basic aqueous solution at room temperature. The reaction solution was stirred and monitored with TLC, and it took 2 hours for the completion of the reaction. Then, concentrated $\mathrm{HCl}$ was gradually added to adjust the $\mathrm{pH}$ to 2.0. The precipitates were collected by filtration, washed with distilled water, and dried to afford the title compound 3. The product was dissolved in methanol and recrystallized by slow evaporation of the solvent in order to generate colorless, bead-like crystals of $N$-(2-ethoxyphenyl) benzenesulfonamide (yield $89 \%$; m.p. $88^{\circ} \mathrm{C}$ ).

\section{General procedure for the synthesis of $\mathrm{N}$-alkyl substituted sulfonamides in DMF}

The calculated amount of $3(0.1 \mathrm{mmol})$ was taken in a round-bottomed flask $(50 \mathrm{~mL})$, and dimethyl formamide (DMF; $10 \mathrm{~mL}$ ) was added to dissolve it; sodium hydride $(0.1 \mathrm{mmol})$ was then added to the mixture. The mixture was stirred for 30 minutes at room temperature, then the alkyl halide was slowly added to the mixture, and the solution was stirred for a further 3 hours. The progress of the reaction was monitored via TLC till single spot. The product was precipitated by adding water. It was filtered, washed with water, and crystallized from aqueous methanol.

\section{General procedure for the synthesis of $\mathrm{N}$-acylated derivatives}

The calculated amount of 1 was taken in the roundbottomed flask $(50 \mathrm{~mL})$, then a small amount of sodium carbonate $\left(\mathrm{Na}_{2} \mathrm{CO}_{3}\right)$ was added to make the medium basic. Acyl chloride ( 8 and 10 separately) was then added, and 
the solution was shaken vigorously. The evolved $\mathrm{CO}_{2}$ was liberated at intervals. The reaction progress was monitored with TLC till single spot. After completion of the reaction, water was added, and the precipitates that were formed were filtered and dried.

\section{Acetylcholinesterase assay}

AChE inhibition activity was performed according to the method of Ellman et al. (1961) with slight modifications. The total volume of the reaction mixture was $100 \mu \mathrm{L}$. It contained $60 \mu \mathrm{L}$ of $\mathrm{Na}_{2} \mathrm{HPO}_{4}$ buffer with a concentration of $50 \mathrm{mM}$ and a $\mathrm{pH}$ of 7.7. Following this, $10 \mu \mathrm{L}$ of the test compound $\left(0.5 \mathrm{mM} \mathrm{well}^{-1}\right)$ was added, followed by the addition of $10 \mu \mathrm{L}\left(0.005\right.$ unit well $\left.^{-1}\right)$ of enzyme. The contents were mixed and preread at $405 \mathrm{~nm}$, then contents were preincubated for 10 minutes at $37^{\circ} \mathrm{C}$. The reaction was initiated by the addition of $10 \mu \mathrm{L}$ of $0.5 \mathrm{mM}$ well $^{-1}$ substrate (acetylthiocholine iodide), followed by the addition of $10 \mu \operatorname{L}$ of DTNB $\left(0.5 \mathrm{mM}^{-1} \mathrm{w}^{-1}\right)$. After 15 minutes of incubation at $37^{\circ} \mathrm{C}$, absorbance was measured at $405 \mathrm{~nm}$. A Synergy HT (BioTek, USA) 96well plate reader was used in all experiments. All experiments were carried out with their respective controls in triplicate. Eserine $\left(0.5 \mathrm{mM}_{\text {well }}{ }^{-1}\right)$ was used as a positive control. The percent of inhibition was calculated using the following equation:

$$
\text { Inhibition }(\%)=\frac{\text { Control }- \text { Test }}{\text { Control }} \times 100
$$

\section{Butyrylcholinesterase assay}

BChE inhibition activity was performed according to the method of Ellman et al. (1961) with slight modifications. The total volume of the reaction mixture was $100 \mu \mathrm{L}$ containing $60 \mu \mathrm{L}$ of the $\mathrm{Na}_{2} \mathrm{HPO}_{4}$ buffer $(50 \mathrm{mM}$ and a $\mathrm{pH}$ of 7.7). A total of $10 \mu \mathrm{L}$ of the test compounds of $0.5 \mathrm{mM}$ well ${ }^{-1}$ were added, followed by the addition of $10 \mu \mathrm{L}\left(0.5\right.$ unit well $\left.^{-1}\right)$ of BChE. The contents were mixed and preread at $405 \mathrm{~nm}$ and then preincubated for 10 minutes at $37^{\circ} \mathrm{C}$. The reaction was initiated by the addition of $10 \mu \mathrm{L}$ of the $0.5 \mathrm{mM}_{\text {well }}{ }^{-1}$ substrate (butyrylthiocholine bromide) followed by the addition of $10 \mu \mathrm{L}$ of DTNB, $0.5 \mathrm{mM}_{\text {well }}{ }^{-1}$. After 15 minutes of incubation at $37^{\circ} \mathrm{C}$, absorbance was measured at $405 \mathrm{~nm}$. A Synergy HT (BioTek, USA) 96-well plate reader was used in all experiments. All experiments were carried out with their respective controls in triplicate. Eserine $\left(0.5 \mathrm{mM}\right.$ well $\left.{ }^{-1}\right)$ was used as a positive control. The percent of inhibition was calculated by the help of following equation:

$$
\text { Inhibition }(\%)=\frac{\text { Control }- \text { Test }}{\text { Control }} \times 100
$$

$\mathrm{IC}_{50}$ values (the concentration at which there is $50 \%$ enzyme inhibition) of the compounds were calculated using the EZ-Fit Enzyme kinetics software (Perella Scientific Inc., Amherst, USA).

\section{Lipoxygenase assay}

LOX activity was assayed according to the reported method (Clapp et al., 1985; Kemal et al., 1987), but with slight modifications. A total volume of $200 \mu \mathrm{L}$ of the assay mixture contained $150 \mu \mathrm{L}$ sodium phosphate buffer (100 mM, pH 8.0), $10 \mu \mathrm{L}$ of the test compound, and $15 \mu \mathrm{L}$ of the purified LOX enzyme (Sigma, USA). The contents were mixed and preread at $234 \mathrm{~nm}$, and then preincubated for 10 minutes at $25^{\circ} \mathrm{C}$. The reaction was initiated by the addition of $25 \mu \mathrm{L}$ of substrate solution. The change in absorbance was observed after 6 minutes at $234 \mathrm{~nm}$. A Synergy HT (BioTek, USA) 96-well plate reader was used in all experiments. All reactions were performed in triplicate. The positive and negative controls were included in the assay. Quercetin $\left(0.5 \mathrm{mM}_{\text {well }}{ }^{-1}\right)$ was used as a positive control. The percentage of inhibition was calculated by the formula given below:

$$
\text { Inhibition }(\%)=\frac{\text { Control }- \text { Test }}{\text { Control }} \times 100
$$

where Control $=$ Total enzyme activity without inhibitor; Test $=$ Activity in the presence of the test compound.

\section{DPPH assay}

The stable 1,1-diphenyl-2-picrylhydrazyl radical (DPPH) was used for the determination of antioxidant activity. Different concentrations of the compounds in their respective solvents were added at an equal volume $(10 \mu \mathrm{L})$ to $90 \mu \mathrm{L}$ of $100 \mu \mathrm{M}$ of methanolic DPPH in a total volume of $100 \mu \mathrm{L}$ in $96-w e l l$ plates. The contents were mixed and incubated at $37^{\circ} \mathrm{C}$ for 30 minutes. The absorbance was measured at $517 \mathrm{~nm}$ using a Synergy HT BioTek ${ }^{\circledR}$ USA microplate reader. Quercetin and $\mathrm{L}$-ascorbic acid were used as standard antioxidants. The experiments were carried out in triplicate. $\mathrm{IC}_{50}$ values were calculated using the EZ-Fit5 (Perrella Scientific Inc., Amherst, USA) software. The decrease in absorbance indicates increased radical scavenging activity, which was determined by the following formula (Huang et al., 2005): 
Antiradical ativity $(\%$ Inhibition $)=\frac{\text { Control }- \text { Test }}{\text { Control }} \times 100$

\section{Structural characterization}

\section{$\mathrm{N}$-(2-Ethoxyphenyl)benzenesulfonamide (3)}

Dark brown beads, yield $89 \%$, m.p. $88^{\circ} \mathrm{C}$; IR (KBr): $v_{\max }: 3430(\mathrm{~N}-\mathrm{H}), 3056(\mathrm{Ar}-\mathrm{H}), 1341\left(-\mathrm{SO}_{2-} \mathrm{NH}-\right), 1258$ (Ar-O-R); EIMS: $m / z 277[\mathrm{M}]^{+}, 232\left[\mathrm{M}-O C_{2} \mathrm{H}_{5}\right]^{+}, 213$ $\left[\mathrm{M}-\mathrm{SO}_{2}\right]^{+}, 141\left[\mathrm{C}_{6} \mathrm{H}_{5} \mathrm{SO}_{2}\right]^{+}$.

N-Methyl-N-(2-ethoxyphenyl)benzenesulfonamide (5a)

Dark brown powder, yield $83 \%$, m.p. $70{ }^{\circ} \mathrm{C}$; IR $(\mathrm{KBr}): v_{\max }: 3466(\mathrm{~N}-\mathrm{R}), 3040(\mathrm{Ar}-\mathrm{H}), 1344\left(-\mathrm{SO}_{2-} \mathrm{NR}-\right)$,

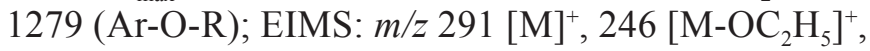
$227\left[\mathrm{M}-\mathrm{SO}_{2}\right]^{+}, 141\left[\mathrm{C}_{6} \mathrm{H}_{5} \mathrm{SO}_{2}\right]^{+}$.

$\mathrm{N}$-Ethyl-N-(2-ethoxyphenyl)benzenesulfonamide (5b)

Dark brown powder, yield $86 \%$, m.p. $54{ }^{\circ} \mathrm{C}$ IR $(\mathrm{KBr}): v_{\max }: 3401(\mathrm{~N}-\mathrm{R}), 3031(\mathrm{Ar}-\mathrm{H}), 1343\left(-\mathrm{SO}_{2-} \mathrm{NR}-\right)$, 1260 (Ar-O-R); EIMS: m/z $305[\mathrm{M}]^{+}, 260\left[\mathrm{M}-\mathrm{OC}_{2} \mathrm{H}_{5}\right]^{+}$, $241\left[\mathrm{M}-\mathrm{SO}_{2}\right]^{+}, 141\left[\mathrm{C}_{6} \mathrm{H}_{5} \mathrm{SO}_{2}\right]^{+}$.

$\mathrm{N}$-Isopropyl-N-(2-ethoxyphenyl)benzenesulfonamide (5C)

Dark brown powder, yield $81 \%$, m.p. $60{ }^{\circ} \mathrm{C}$. IR $(\mathrm{KBr}): v_{\max }: 3432(\mathrm{~N}-\mathrm{R}), 3075(\mathrm{Ar}-\mathrm{H}), 1323\left(-\mathrm{SO}_{2-} \mathrm{NR}-\right)$,

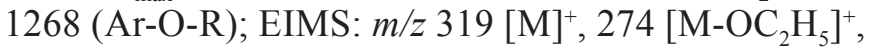
$255\left[\mathrm{M}-\mathrm{SO}_{2}\right]^{+}, 141\left[\mathrm{C}_{6} \mathrm{H}_{5} \mathrm{SO}_{2}\right]^{+}$.

N-Propyl-N-(2-ethoxyphenyl)benzenesulfonamide (5d)

Dark brown powder, yield $89 \%$, m.p. $56{ }^{\circ} \mathrm{C}$ IR $(\mathrm{KBr}): v_{\max }: 3467$ (N-R), 3065 (Ar-H), 1343 (-SO $\left.\mathrm{SO}_{2} \mathrm{NR}-\right)$

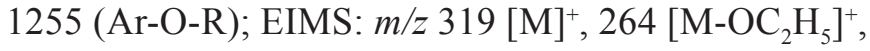
$255\left[\mathrm{M}-\mathrm{SO}_{2}\right]^{+}, 141\left[\mathrm{C}_{6} \mathrm{H}_{5} \mathrm{SO}_{2}\right]^{+}$.

$\mathrm{N}$-Butyl-N-(2-ethoxyphenyl) benzenesulfonamide (5e)

Dark brown powder, yield $82 \%$, m.p. $59{ }^{\circ} \mathrm{C}$. IR $(\mathrm{KBr}): v_{\max }: 3484(\mathrm{~N}-\mathrm{R}), 3049(\mathrm{Ar}-\mathrm{H}), 1315\left(-\mathrm{SO}_{2-} \mathrm{NR}-\right)$,

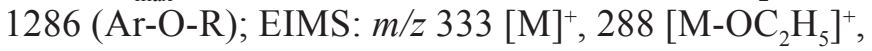
$269\left[\mathrm{M}-\mathrm{SO}_{2}\right]^{+}, 141\left[\mathrm{C}_{6} \mathrm{H}_{5} \mathrm{SO}_{2}\right]^{+}$.

N-Pentyl-N-(2-ethoxyphenyl)benzenesulfonamide (5f)

Dark brown powder, yield $84 \%$, m.p. $58{ }^{\circ} \mathrm{C}$. IR $(\mathrm{KBr}): v_{\max }: 3452(\mathrm{~N}-\mathrm{R}), 3063(\mathrm{Ar}-\mathrm{H}), 1311\left(-\mathrm{SO}_{2-} \mathrm{NR}-\right)$, 1263 (Ar-O-R); EIMS: m/z $347\left[\text { M }^{+}, 302 \text { [M-OC }_{2} \mathrm{H}_{5}\right]^{+}$, $283\left[\mathrm{M}-\mathrm{SO}_{2}\right]^{+}, 141\left[\mathrm{C}_{6} \mathrm{H}_{5} \mathrm{SO}_{2}\right]^{+}$.

$\mathrm{N}$-Allyl-N-(2-ethoxyphenyl)benzenesulfonamide (5g)

Gummy solid, yield $80 \%$. IR (KBr): $v_{\max }: 3488$

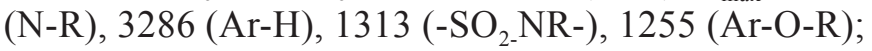

EIMS: $m / z 317[\mathrm{M}]^{+}, 272\left[\mathrm{M}-\mathrm{OC}_{2} \mathrm{H}_{5}\right]^{+}, 253\left[\mathrm{M}-\mathrm{SO}_{2}\right]^{+}$, $141\left[\mathrm{C}_{6} \mathrm{H}_{5} \mathrm{SO}_{2}\right]^{+}$.

\section{N-Benzoyl-2-ethoxyaniline (7)}

Gummy solid, yield 83\%. IR (KBr): $v_{\max }: 3429(\mathrm{~N}-$ H), 3067 (Ar-H), 1253 (Ar-O-R); EIMS: $m / z 241[\mathrm{M}]^{+}$, $196\left[\mathrm{M}-\mathrm{OC}_{2} \mathrm{H}_{5}\right]^{+}, 213[\mathrm{M}-\mathrm{CO}]^{+}, 105\left[\mathrm{C}_{6} \mathrm{H}_{5} \mathrm{CO}\right]^{+}$.

\section{$\mathrm{N}$-Acetyl-2-ethoxyaniline (9)}

Gummy solid, yield 87\%. IR (KBr): $v_{\max }: 3424(\mathrm{~N}-$ H), 3034 (Ar-H), 1244 (Ar-O-R); EIMS: $m / z 179$ [M] , $134\left[\mathrm{M}-\mathrm{OC}_{2} \mathrm{H}_{5}\right]^{+}, 151[\mathrm{M}-\mathrm{CO}]^{+}, 43\left[\mathrm{CH}_{3} \mathrm{CO}\right]^{+}$.
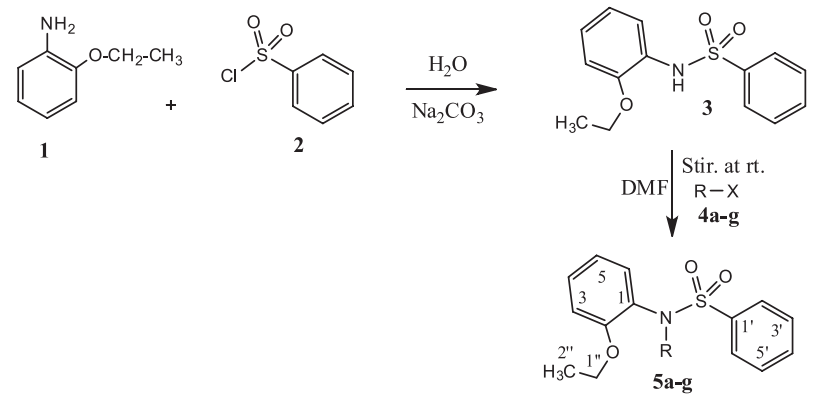
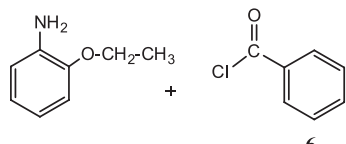

1

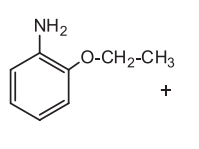

1

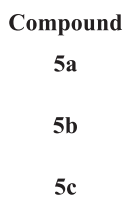

$5 d$

$$
\begin{gathered}
-\mathrm{CH}_{2}-\mathrm{CH}_{2}-\mathrm{CH}_{3} \\
1^{\prime \prime \prime} \quad 2^{\prime \prime} \quad 3^{\prime \prime \prime}
\end{gathered}
$$

FIGURE 1 - Synthesis of 2-phenitidine derivatives 5a-g, 7, and 9.

\section{RESULTS AND DISCUSSION}

The designed $N$-substituted new derivatives of 2-phenitidine were synthesized according to Figure 1. The parent compound $\mathbf{3}$ was synthesized as dark brown beads. The molecular formula, $\mathrm{C}_{14} \mathrm{H}_{15} \mathrm{NO}_{3} \mathrm{~S}$, was established with the help of EI-MS showing a molecular ion peak at $m / z 277$, and by counting the number of protons in the ${ }^{1} \mathrm{H}-\mathrm{NMR}$ spectrum (Table-I). Its IR spectrum revealed the presence of an ethoxy group $\left(1258 \mathrm{~cm}^{-1}\right)$, a sulfonyl group 
TABLE I - ${ }^{1} \mathrm{H}-\mathrm{NMR}{ }^{\mathrm{a}}$ data $\left(\mathrm{CDCl}_{3}\right)$ of compounds $\mathbf{3}, \mathbf{5 a - g}, \mathbf{7}, \mathbf{9}$. $\delta$ in ppm, $J$ in $\mathrm{Hz}$

\begin{tabular}{|c|c|c|c|c|c|c|c|c|c|c|}
\hline \multirow[t]{2}{*}{ C.No. } & 3 & $5 a$ & $5 b$ & $5 c$ & $5 d$ & $5 e$ & $5 f$ & $5 \mathrm{~g}$ & 7 & 9 \\
\hline & $\delta(\mathbf{H})$ & $\delta(\mathbf{H})$ & $\delta(\mathbf{H})$ & $\delta(\mathbf{H})$ & $\delta(\mathbf{H})$ & $\delta(\mathbf{H})$ & $\delta(\mathbf{H})$ & $\delta(\mathbf{H})$ & $\delta(\mathbf{H})$ & $\delta(\mathbf{H})$ \\
\hline 1 & - & - & - & - & - & - & - & - & - & - \\
\hline 3 & $\begin{array}{c}6.67(\mathrm{dd}, \\
J=1.0,7.6)\end{array}$ & $\begin{array}{c}6.74(\mathrm{dd} \\
J=1.0,8.0)\end{array}$ & $\begin{array}{c}6.74(\mathrm{dd}, \\
J=1.2,8.4)\end{array}$ & $\begin{array}{c}6.68(\mathrm{dd}, \\
J=1.0,8.0)\end{array}$ & $\begin{array}{c}6.73(\mathrm{dd}, \\
J=1.0,8.4)\end{array}$ & $\begin{array}{c}6.72(\mathrm{dd} \\
J=1.0,8.4)\end{array}$ & $\begin{array}{c}6.74(\mathrm{dd}, \\
J=1.0,8.4)\end{array}$ & $\begin{array}{c}6.8(\mathrm{dd}, \\
J=1.0,8.0)\end{array}$ & $\begin{array}{c}6.88(\mathrm{dd}, \\
J=1.2,7.6)\end{array}$ & $\begin{array}{c}6.84(\mathrm{dd}, \\
J=1.0,7.6)\end{array}$ \\
\hline 5 & $\begin{array}{c}6.87 \text { (ddd, } \\
J=1.2,7.6, \\
8.0)\end{array}$ & $\begin{array}{c}6.91(\mathrm{ddd}, \\
J=1.6,8.0, \\
8.0)\end{array}$ & $\begin{array}{c}6.91(\mathrm{ddd}, \\
J=1.2,7.6,7.6)\end{array}$ & $\begin{array}{c}6.89(\mathrm{ddd} \\
J=1.2,7.6,7.6)\end{array}$ & $\begin{array}{c}6.91(\mathrm{ddd}, \\
J=1.2,7.6,7.6)\end{array}$ & $\begin{array}{c}6.91 \text { (ddd, } \\
J=1.07 .2 \\
7.6)\end{array}$ & $\begin{array}{c}6.91(\mathrm{ddd}, \\
J=1.2,7.6, \\
7.6)\end{array}$ & $\begin{array}{c}6.87(\mathrm{ddd}, \\
J=1.2,7.6,8.4)\end{array}$ & $\begin{array}{c}6.99 \text { (ddd, } \\
J=1.6,7.6, \\
7.6)\end{array}$ & $\begin{array}{c}6.91 \text { (ddd, } \\
J=1.2,7.6, \\
8.0)\end{array}$ \\
\hline 6 & $\begin{array}{c}7.52(\mathrm{dd}, \\
J=1.2,7.6)\end{array}$ & $\begin{array}{c}7.36(\mathrm{dd}, J= \\
2.0,8.0)\end{array}$ & $\begin{array}{c}7.31(\mathrm{dd}, \\
J=1.6,7.6)\end{array}$ & $\begin{array}{c}7.13(\mathrm{dd}, \\
J=1.6,7.6)\end{array}$ & $\begin{array}{c}7.30(\mathrm{dd}, \\
J=2.0,8.0)\end{array}$ & $\begin{array}{l}7.31(\mathrm{dd} \\
J=2,8.0)\end{array}$ & $\begin{array}{c}7.32(\mathrm{dd}, \\
J=1.6,7.6)\end{array}$ & $\begin{array}{c}7.27(\mathrm{dd}, \\
J=1.6,7.6)\end{array}$ & $\begin{array}{c}8.52(\mathrm{dd}, \\
J=1.6,7.6)\end{array}$ & $\begin{array}{c}8.33 \text { (br d } \\
J=7.6)\end{array}$ \\
\hline $3^{\prime}$ & $\begin{array}{c}7.36 \text { (br t } \\
J=7.6)\end{array}$ & $\begin{array}{l}7.41 \text { (br t } \\
J=7.6)\end{array}$ & $\begin{array}{c}7.38 \text { (br t } \\
J=7.2 \text { ) }\end{array}$ & $\begin{array}{c}7.41 \text { (br t } \\
J=7.2 \text { ) }\end{array}$ & $\begin{array}{c}7.38(\mathrm{br} \mathrm{t} \\
J=8.0)\end{array}$ & $\begin{array}{c}7.39 \text { (br t } \\
J=7.6)\end{array}$ & $\begin{array}{c}7.38 \text { (br.t, } \\
J=7.6)\end{array}$ & $\begin{array}{c}7.38(\mathrm{br} \mathrm{t} \\
J=7.2)\end{array}$ & $\begin{array}{c}7.45(\mathrm{br} \mathrm{t} \\
J=8.6)\end{array}$ & - \\
\hline $4^{\prime}$ & $\begin{array}{c}7.47 \text { (br t } \\
J=7.6)\end{array}$ & $\begin{array}{c}7.51 \text { (br t } \\
J=7.6)\end{array}$ & $\begin{array}{c}7.48 \text { (br t } \\
J=7.6)\end{array}$ & $\begin{array}{c}7.49 \text { (br t } \\
J=7.6)\end{array}$ & $\begin{array}{c}7.48(\mathrm{br} \mathrm{t} \\
J=7.6)\end{array}$ & $\begin{array}{c}7.49 \text { (br t } \\
J=7.6)\end{array}$ & $\begin{array}{c}7.49 \text { (br.t. } \\
J=7.6)\end{array}$ & $\begin{array}{c}7.48 \text { (br t } \\
J=7.6)\end{array}$ & $\begin{array}{c}7.54(\mathrm{br} \mathrm{t} \\
J=8.2)\end{array}$ & - \\
\hline $5^{\prime}$ & $\begin{array}{c}7.36 \text { (br t } \\
J=7.6)\end{array}$ & $\begin{array}{l}7.41 \text { (br t } \\
J=7.6)\end{array}$ & $\begin{array}{c}7.38 \text { (br t } \\
J=7.2)\end{array}$ & $\begin{array}{l}7.41(\mathrm{br} \mathrm{t} \\
J=7.2)\end{array}$ & $\begin{array}{c}7.38 \text { (br t } \\
J=8.0)\end{array}$ & $\begin{array}{c}7.39 \text { (br t } \\
J=7.6)\end{array}$ & $\begin{array}{c}7.38 \text { (br.t, } \\
J=7.6)\end{array}$ & $\begin{array}{c}7.38 \text { (br t } \\
J=7.2 \text { ) }\end{array}$ & $\begin{array}{c}7.45 \text { (br t } \\
J=8.6)\end{array}$ & - \\
\hline $6^{\prime}$ & $\begin{array}{c}7.71(\mathrm{dd}, \\
J=1.6,8.8)\end{array}$ & $\begin{array}{c}7.66(\mathrm{dd}, \\
J=1.6,8.8)\end{array}$ & $\begin{array}{c}7.66(\mathrm{dd}, \\
J=1.2,8.4)\end{array}$ & $\begin{array}{c}7.82(\mathrm{dd}, \\
J=1.2,8.4)\end{array}$ & $\begin{array}{c}7.63(\mathrm{dd}, \\
J=1.2,7.2)\end{array}$ & $\begin{array}{c}7.63(\mathrm{dd}, \\
J=1.2,7.2)\end{array}$ & $\begin{array}{c}7.63(\mathrm{dd}, \\
J=1.2,8.8)\end{array}$ & $\begin{array}{c}7.63(\mathrm{dd}, \\
J=1.2,8.4)\end{array}$ & $\begin{array}{c}7.88(\mathrm{dd}, \\
J=1.6,8.4)\end{array}$ & - \\
\hline 1" & $\begin{array}{l}3.78(\mathrm{q}, \\
J=7.2)\end{array}$ & $\begin{array}{l}3.64(\mathrm{q} \\
J=6.8)\end{array}$ & $3.64(\mathrm{~m}, 2 \mathrm{H})$ & $\begin{array}{l}3.83(\mathrm{q}, \\
J=6.8)\end{array}$ & $3.59(\mathrm{~m}, 4 \mathrm{H})$ & $\begin{array}{c}3.59(\mathrm{~m}, 2 \mathrm{H}, \\
\left.\mathrm{CH} 2-1^{\prime \prime}\right),\end{array}$ & $3.59(\mathrm{~m})$ & $4.20(\mathrm{~m})$ & $\begin{array}{l}4.14(\mathrm{q}, \\
J=6.8)\end{array}$ & - \\
\hline 4"' & - & - & - & - & - & $\begin{array}{l}0.83(\mathrm{t}, \\
J=7.2)\end{array}$ & $1.24(\mathrm{~m})$ & - & - & - \\
\hline 5"' & - & - & - & - & - & & $\begin{array}{l}0.82(\mathrm{t}, \\
J=7.2)\end{array}$ & - & - & - \\
\hline
\end{tabular}

${ }^{\text {a }}$ All spectra were recorded at $400 \mathrm{MHz}$.

$\left(1341 \mathrm{~cm}^{-1}\right)$, and a-NH group $\left(3430 \mathrm{~cm}^{-1}\right)$ in the molecule. The EI-MS also gave a distinct peak at $\mathrm{m} / \mathrm{z} 213$ after the removal of the $-\mathrm{SO}_{2}$ group, and a further two fragments of ion peaks were observed at $\mathrm{m} / \mathrm{z} 232\left[\mathrm{M}-\mathrm{OC}_{2} \mathrm{H}_{5}\right]^{+}$and 141 $\left[\mathrm{C}_{6} \mathrm{H}_{5} \mathrm{SO}_{2}\right]^{+}$, which showed the presence of ethoxy and benzene sulfonyl groups, respectively, in the molecule. In its ${ }^{1} \mathrm{H}-\mathrm{NMR}$ spectrum, the signals in the aromatic region appeared at $\delta 7.52$ (dd, $J=1.2,7.6 \mathrm{~Hz}, \mathrm{H}-6), 7.00$ (ddd, $J$
$=1.2,7.6,8.0 \mathrm{~Hz}, \mathrm{H}-4), 6.87$ (ddd, $J=1.2,7.6,8.0 \mathrm{~Hz}, \mathrm{H}-5)$, and $6.67(\mathrm{dd}, J=1.0,7.6 \mathrm{~Hz}, \mathrm{H}-3)$, which were assigned to the protons of the disubstituted ring of phenitidine, and the signals appearing at $\delta 7.71\left(\mathrm{dd}, J=1.6,8.8 \mathrm{~Hz}, 2 \mathrm{H}, \mathrm{H}-2^{\prime}\right.$ \& H-6'), 7.47 (brt, $J=7.6 \mathrm{~Hz}, \mathrm{H}-4^{\prime}$ ), and 7.36 (br t, $J=7.6$ $\left.\mathrm{Hz}, \mathrm{H}-3^{\prime} \& \mathrm{H}-5^{\prime}\right)$ were typical for the protons of the monosubstituted ring derived from benzenesulphonyl chloride. Moreover, a characteristics quartet at $\delta 3.78(\mathrm{q}, J=7.2 \mathrm{~Hz}$, 
TABLE II - Enzyme inhibition studies of 2-phenitidine derivatives $(n=3$, mean \pm SEM)

\begin{tabular}{lcccccccc}
\hline \multirow{2}{*}{$\begin{array}{l}\text { Compound } \\
\text { No. }\end{array}$} & \multicolumn{2}{c}{ DPPH } & \multicolumn{2}{c}{ AChE } & \multicolumn{2}{c}{ BChE } & \multicolumn{2}{c}{ LOX } \\
\cline { 2 - 9 } & at $0.5 \mathrm{mM}$ & $\mu$ moles & at $0.5 \mathrm{mM}$ & $\begin{array}{c}\left(\mathrm{IC}_{50}\right) \\
\mu \text { moles }\end{array}$ & $\begin{array}{c}(\%) \\
\text { at } 0.5 \mathrm{mM}\end{array}$ & $\begin{array}{c}\left(\mathrm{IC}_{50}\right) \\
\mu \text { moles }\end{array}$ & $\begin{array}{c}(\%) \\
\text { at } 0.5 \mathrm{mM}\end{array}$ & $\begin{array}{c}\left(\mathrm{IC}_{50}\right) \\
\mu \mathrm{moles}\end{array}$ \\
\hline $\mathbf{3}$ & $9.10 \pm 0.59$ & Nil & $38.44 \pm 0.47$ & Nil & $72.05 \pm 0.98$ & $68 \pm 0.99$ & $13.48 \pm 0.66$ & Nil \\
$\mathbf{5 a}$ & $14.66 \pm 0.78$ & Nil & $40.39 \pm 0.95$ & Nil & $81.84 \pm 0.87$ & $132 \pm 1.01$ & $4.24 \pm 0.45$ & Nil \\
$\mathbf{5 b}$ & $14.59 \pm 0.75$ & Nil & $50.65 \pm 0.83$ & $>500$ & $82.18 \pm 0.45$ & $10 \pm 0.85$ & $4.02 \pm 1.06$ & Nil \\
$\mathbf{5 c}$ & $17.86 \pm 0.91$ & Nil & $37.79 \pm 1.01$ & Nil & $90.51 \pm 1.02$ & $07 \pm 0.81$ & $3.70 \pm 0.92$ & Nil \\
$\mathbf{5 d}$ & $25.30 \pm 0.84$ & Nil & $36.49 \pm 0.93$ & Nil & $78.14 \pm 1.11$ & $72 \pm 1.00$ & $15.87 \pm 0.72$ & Nil \\
$\mathbf{5 e}$ & $27.87 \pm 0.73$ & Nil & $34.68 \pm 0.89$ & Nil & $69.67 \pm 0.98$ & $131 \pm 0.69$ & $18.48 \pm 0.76$ & Nil \\
$\mathbf{5 f}$ & $46.56 \pm 1.02$ & Nil & $36.10 \pm 0.77$ & Nil & $66.67 \pm 0.65$ & $169 \pm 0.77$ & $10.54 \pm 0.84$ & Nil \\
$\mathbf{5 g}$ & $16.89 \pm 0.59$ & Nil & $47.01 \pm 0.84$ & Nil & $83.35 \pm 0.59$ & $78 \pm 0.78$ & $13.59 \pm 0.69$ & Nil \\
$\mathbf{7}$ & $22.86 \pm 0.68$ & Nil & $30.65 \pm 0.91$ & Nil & $77.60 \pm 0.48$ & $141 \pm 1.03$ & $59.78 \pm 0.93$ & $257 \pm 0.88$ \\
$\mathbf{9}$ & $10.42 \pm 1.01$ & Nil & $31.95 \pm 1.00$ & Nil & $60.81 \pm 0.98$ & $298 \pm 1.10$ & $13.26 \pm 0.85$ & Nil \\
Control & Quercetin & $16.96 \pm 0.14$ & Eserine & $0.04 \pm 0.001$ & Eserine & $0.85 \pm 0.001$ & Quercetin & $37.12 \pm 0.07$ \\
\hline
\end{tabular}

Note: DPPH = 1,1-diphenyl-2-picrylhydrazyl radical

$2 \mathrm{H}, \mathrm{H}_{2}-1$ ") and a triplet at $\delta 1.22\left(\mathrm{t}, J=7.2 \mathrm{~Hz}, 3 \mathrm{H}, \mathrm{H}_{3}-2\right.$ ") revealed the presence of an ethoxy group in the molecule. On the basis of the cumulative evidence reported above, the structure of $\mathbf{3}$ was assigned as $N$-(2-ethoxyphenyl) benzenesulfonamide, which is a new sulfonamide. Similarly, on the basis of spectral evidences from IR, EI-MS (experimental section) and, ${ }^{1} \mathrm{H}-\mathrm{NMR}$ (Table I), the structures of other derivatives of 2-phenitidine were elucidated as described in Figure 1.

The screening of these derivatives showed that these were inactive against LOX and AChE, but they exhibited moderate inhibitory potential against $\mathrm{BChE}$, as evident from their $\mathrm{IC}_{50}$ values (Table II). Among these, $\mathrm{N}$ isopropyl- $N$-(2-ethoxyphenyl) benzenesulfonamide (5c) was found to be the most suitable inhibitor, having an $\mathrm{IC}_{50}$ value of $07 \pm 0.81 \mu \mathrm{moles} / \mathrm{L}$ relative to eserine, a reference standard, with an $\mathrm{IC}_{50}$ value of $0.85 \pm 0.001 \mu$ moles/L, probably due to the substitution of the isopropyl group (a branched chain) in this molecule. Antioxidant activity using the DPPH method showed little antioxidant activity of these compounds; the highest activity was shown by $5 \mathrm{f}$ $(46.56 \% \pm 1.02 \%$ at $0.5 \mathrm{mM})$ and the lowest activity by parent compound $3(9.10 \% \pm 0.59 \%$ at $0.5 \mathrm{mM})$. All other compounds showed antioxidant activity between these two extremes. The present investigation generally concluded that the synthesized 2-phenitidine derivatives, 5a-5g, 7 , and 9 - due to their suitable structural features - could be used as valuable inhibitors of BChEs, and that these are also ideally suited for further structural modification to obtain more potent and less cytotoxic therapeutic agents for the treatment of Alzheimer's disease.

\section{REFERENCES}

BERTACCINI, G. (Ed.). Substance P. In: Handbook of experimental pharmacology. Berlin: Springer, 1982. v.59/ II, p. 85-105.

BORUAH, A.; BARUAH, M.; PRAJAPATI, D.; SANDHU J.S. The efficient chemoselective reduction of azides to primary amines. Synlett, v.11, p.1253-1254, 1997.

CHAN, W.Y.; BERTHELETTE, C. A mild, efficient method for the synthesis of aromatic and aliphatic sulfonamides. Tetrahedron Lett., v.43, p.4537-4540, 2002.

CLAPP, H.C.; BANERJEE, A.; ROTENBERG, S.A. Inhibition of soybean lipoxygenase 1 by $n$-alkylhydroxylamines. $J$. Biochem., v.24, p.1826-1830, 1985.

CYGLER, M.; SCHRAG, J.D.; SUSSMAN J.; HAREL, L.M.; SILMAN, I.; GENTRY, M.K. Relationship between sequence conservation and three-dimensional structure in a large family of esterases, lipases and related proteins. Protein Sci., v.2, p.366-382, 1993.

DOWNER-RILEY, N.K., JACKSON, Y.A. Iodine-mediated cyclisation of thiobenzamides to produce benzothiazoles and benzoxazoles. Tetrahedron, v.63, p.10276-10281, 2007. 
ELLMAN, G.L.; COURTNEY, K.D.; ANDRES JR., V.; FEATHERSTONE, R.M. A new and rapid calorimetric determination of acetylcholinesterase activity. Biochem. Pharmacol., v.7, p.88-90, 1961.

GARAJ, V.; PUCCETTI, L.; FASOLIS, G.; WINUM, J.Y.J.; MONTERO, L.; SCOZZAFAVA, A.; VUlLO, D.; INNOCENTI, A.; SUPURAN, C.T. Carbonic anhydrase inhibitors: novel sulfonamides incorporating 1,3,5-triazine moieties as inhibitors of the cytosolic and tumour-associated carbonic anhydrase isozymes I, II and IX. Bioorg. Med. Chem. Lett., v.15, p.3102-3108, 2005.

GAUTHIER, S. Cholinergic adverse effects of cholinesterase inhibitors in Alzheimer's disease. Drug. Aging., v.18, p.853$862,2001$.

HUANG, D.J.; CHEN, H.J.; LIN, C.D.; LIN, Y.H. Antioxidant and antiproliferative activities of water spinach (Ipomoea aquatic Forsk) constituents. Bot. Bull. Acad. Sin., v.46, p.99-106, 2005.

KEMAL, C.; LOUIS-FLEMBERG, P.; KRUPINSKI-OLSEN, R. SHORTER, A.L. Reproductive inactivation of soybean lipoxygenase activity. J. Biochem., v.26, p.7064-7072, 1987.

KIVELA, A.J.; KIVELA, J.; SAARNIO, J.; PARKKILA, S. Carbonic anhydrases in normal gastrointestinal tumours. World J. Gastro., v.11, p.155-163, 2005.

REMKO, M.; KOZISEK, J.; SEMANOVA, J.; GREGAN, F. Synthesis, crystal and molecular structure of two biologically active aromatic sulfonamides and their hydrochloride salts. J. Mol. Struct., v.973, p.18-20, 2010.

SACZEWSKI, F.; INNOCENTI, A.; SLAWINSKI, J.; KORNICKA, A.; BRZOZOWSKI, Z.; POMARNACKA, E.L.; SCOZZAFAVA, A.; TEMPERINI, C.; SUPURAN, C.T. Carbonic anhydrase inhibitors: Inhibition of human cytosolic isozymes I and II and tumor-associated isozymes IX and XII with S-substituted 4-chloro-2-mercapto-5methyl-benzenesulfonamide. Bioorg. Med. Chem., v.16, p.3933-3940, 2008.
SACZEWSKI, F.; SLAWINSKI, J.; KORNICKA, A.; BRZOZOWSKI, Z.; POMARNACKA, E.; INNOCENTI, A.; SCOZZAFAVA, A.; SUPURAN, C.T. Carbonic anhydrase inhibitors. Inhibition of the cytosolic human isozymes I and II, and the transmembrane, tumorassociated isozymes IX and XII with substituted aromatic sulfonamides activatable in hypoxic tumors. Bioorg. Med. Chem. Lett., v.16, p.4846-4851, 2006.

SHAABANI, A.; SOLEIMANI, E.; REZAYAN, A.H. A novel approach for the synthesis of alkyl and aryl sulfonamides. Tetrahedron Lett., v.48, p.2185-2188, 2007.

SMITH, K.; EL-HITI, A.G.; JAYNE, J.A.; BUTTERS, M. Acetylation of aromatic ethers using acetic anhydride over solid acid catalysts in a solvent-free system. Org. Biomol. Chem., v.1, p.1560-1564, 2003.

SUPURAN, C.T.; SCOZZAFAVA, A.; CASINI, A. Carbonic anhydrase inhibitors. Med. Res. Rev., v.23, p.146-189, 2003.

SUPURAN, C.T. Carbonic anhydrases: catalytic and inhibition mechanisms, distribution and physiological roles. In: SUPURAN, C.T.; SCOZZAFAVA, A.; CONWAY, J. (Eds.). Carbonic anhydrase: its inhibitors and activators. Boca Raton: CRC Press, 2004. p.1-23.

SUPURAN, C.T.; SCOZZAFAVA, A. Carbonic anhydrases as targets for medicinal chemistry Bioorg. Med. Chem., v.15, p.4336-4350, 2007.

TOUGU, V. Acetylcholinesterase: Mechanism of catalysis and inhibition. Curr. Med. Chem., v.1, p.155-170, 2001.

Received for publication on $10^{\text {th }}$ August 2011 Accepted for publication on $11^{\text {th }}$ October 2012 
